\title{
Montgomery Identity and Ostrowski Type Inequalities for Riemann-Liouville Fractional Integral
}

\author{
Andrea Aglić Aljinović \\ Faculty of Electrical Engineering and Computing, University of Zagreb, Unska 3, 10000 Zagreb, Croatia \\ Correspondence should be addressed to Andrea Aglić Aljinović; andrea.aglic@fer.hr \\ Received 31 May 2014; Accepted 27 August 2014; Published 10 September 2014 \\ Academic Editor: Ralf Meyer
}

Copyright ( 2014 Andrea Aglić Aljinović. This is an open access article distributed under the Creative Commons Attribution License, which permits unrestricted use, distribution, and reproduction in any medium, provided the original work is properly cited.

We present Montgomery identity for Riemann-Liouville fractional integral as well as for fractional integral of a function $f$ with respect to another function $g$. We further use them to obtain Ostrowski type inequalities involving functions whose first derivatives belong to $L_{p}$ spaces. These inequalities are generally sharp in case $p>1$ and the best possible in case $p=1$. Application for Hadamard fractional integrals is given.

\section{Introduction}

The following Ostrowski inequality is well known [1]:

$$
\begin{aligned}
f(x) & -\frac{1}{b-a} \int_{a}^{b} f(t) d t \\
& \leq\left[\frac{1}{4}+\frac{(x-((a+b) / 2))^{2}}{(b-a)^{2}}\right](b-a)\left\|f^{\prime}\right\|_{\infty} .
\end{aligned}
$$

It holds for every $x \in[a, b]$ whenever $f:[a, b] \rightarrow$ $\mathbb{R}$ is continuous on $[a, b]$ and differentiable on $(a, b)$ with derivative $f^{\prime}:(a, b) \rightarrow \mathbb{R}$ bounded on $(a, b)$; that is,

$$
\left\|f^{\prime}\right\|_{\infty}:=\sup _{t \in(a, b)}\left|f^{\prime}(t)\right|<+\infty .
$$

Ostrowski proved this inequality in 1938, and since then it has been generalized in a number of ways. Over the last few decades, some new inequalities of this type have been intensively considered together with their applications in numerical analysis, probability, information theory, and so forth. This inequality can easily be proved by using the following Montgomery identity (see, for instance, [2]):

$$
f(x)=\frac{1}{b-a} \int_{a}^{b} f(t) d t+\int_{a}^{b} P(x, t) f^{\prime}(t) d t,
$$

where $P(x, t)$ is the Peano kernel, defined by

$$
P(x, t)= \begin{cases}\frac{t-a}{b-a}, & a \leq t \leq x, \\ \frac{t-b}{b-a}, & x<t \leq b .\end{cases}
$$

The Riemann-Liouville fractional integral of order $\alpha>0$ is defined by

$$
{ }_{a} J_{x}^{\alpha} f(x)=\frac{1}{\Gamma(\alpha)} \int_{a}^{x}(x-t)^{\alpha-1} f(t) d t
$$

where $\Gamma(\alpha)$ is a gamma function

$$
\Gamma(\alpha)=\int_{0}^{\infty} e^{-t} t^{\alpha-1} d t .
$$

When $a=0$ (5) is the Riemann definition of fractional integral. In case $\alpha=1$, fractional integral reduces to classical integral. 
In [3], the following Montgomery identity for fractional integrals is obtained.

Theorem 1. Let $\alpha \geq 1, f:[a, b] \rightarrow \mathbb{R}$ differentiable on $[a, b]$, $f^{\prime}:[a, b] \rightarrow \mathbb{R}$ integrable on $[a, b]$, and $x \in[a, b]$. Then, the following identity holds:

$$
\begin{aligned}
f(x)= & \frac{\Gamma(\alpha)}{(b-a)}(b-x)^{1-\alpha}{ }_{a} J_{b}^{\alpha} f(b) \\
& -{ }_{a} J_{b}^{\alpha-1}\left(P_{2}(x, b) f(b)\right)+{ }_{a} J_{b}^{\alpha}\left(P_{2}(x, b) f^{\prime}(b)\right),
\end{aligned}
$$

where

$$
P_{2}(x, t)= \begin{cases}\frac{t-a}{b-a}(b-x)^{1-\alpha} \Gamma(\alpha), & a \leq t \leq x, \\ \frac{t-b}{b-a}(b-x)^{1-\alpha} \Gamma(\alpha), & x<t \leq b .\end{cases}
$$

In [3], the authors used this identity to obtain the following Ostrowski type inequality for fractional integrals.

Theorem 2. Let $\alpha \geq 1, f:[a, b] \rightarrow \mathbb{R}$ differentiable on $[a, b]$, and $\left|f^{\prime}(x)\right|<M$ for every $x \in[a, b]$; then, the following Ostrowski inequality holds:

$$
\begin{gathered}
\left|f(x)-\frac{\Gamma(\alpha)}{(b-a)}(b-x)^{1-\alpha}{ }_{a} J_{b}^{\alpha} f(b)+{ }_{a} J_{b}^{\alpha-1}\left(P_{2}(x, b) f(b)\right)\right| \\
\leq \frac{M}{\alpha(\alpha+1)}\left[(b-x)\left(2 \alpha\left(\frac{b-x}{b-a}\right)-\alpha-1\right)\right. \\
\left.+(b-a)^{\alpha}(b-x)^{1-\alpha}\right] .
\end{gathered}
$$

These results were further generalized in [4], while in [5] generalizations are obtained for fractional integral of a function $f$ with respect to another function $g$ (defined in Section 3).

In the present paper, we give another, simpler new generalization of Montgomery identity for Riemann-Liouville fractional integral of order $\alpha$, which holds for a larger set of $\alpha$; that is, $\alpha>0$. We also obtain Montgomery identity for fractional integral of a function $f$ with respect to another function $g$. We further use these identities to obtain generalizations of Ostrowski inequality for fractional integrals of a function $f$ with respect to another function $g$ for functions whose first derivatives $f^{\prime}$ belong to $L_{p}$ spaces. These inequalities are generally sharp in case $p>1$ and the best possible in case $p=1$. As a special case, application for Hadamard fractional integrals is given.

\section{Montgomery Identity for Fractional Integrals}

In this section we give another, simpler new generalization of Montgomery identity for fractional integrals by using the weighted Montgomery identity.
The weighted Montgomery identity (obtained by Pečarić in $[6])$ states that for any $x \in[a, b]$

$$
f(x)=\int_{a}^{b} w(t) f(t) d t+\int_{a}^{b} P_{w}(x, t) f^{\prime}(t) d t,
$$

where $f:[a, b] \rightarrow \mathbb{R}$ is differentiable on $[a, b], f^{\prime}:[a, b] \rightarrow$ $\mathbb{R}$ is integrable on $[a, b], w:[a, b] \rightarrow[0, \infty)$ is some normalized weighted function, that is, integrable function satisfying $\int_{a}^{b} w(t) d t=1, W(t)=\int_{a}^{t} w(x) d x$ for $t \in[a, b]$, and the weighted Peano kernel is

$$
P_{w}(x, t)= \begin{cases}W(t), & a \leq t \leq x \\ W(t)-1 & x<t \leq b .\end{cases}
$$

For the uniform normalized weighted function $w(t)=$ $1 /(b-a), t \in[a, b]$, it reduces to Montgomery identity (3).

Theorem 3. Let $\alpha>0, f:[a, b] \rightarrow \mathbb{R}$ differentiable on $[a, b]$, $f^{\prime}:[a, b] \rightarrow \mathbb{R}$ integrable on $[a, b]$, and $x \in[a, b]$. Then, the following identity holds:

$$
f(x)=\frac{\Gamma(\alpha+1)}{(b-a)^{\alpha}}{ }_{a} J_{b}^{\alpha} f(b)+\int_{a}^{b} P_{3}(x, t) f^{\prime}(t) d t,
$$

where

$$
P_{3}(x, t)= \begin{cases}1-\left(\frac{b-t}{b-a}\right)^{\alpha} & a \leq t \leq x, \\ -\left(\frac{b-t}{b-a}\right)^{\alpha} & x<t \leq b,\end{cases}
$$

and ${ }_{a} J_{x}^{\alpha} f(x)$ is the Riemann-Liouville integral operator of order $\alpha>0$ defined by (5).

Proof. Apply (10) with normalized weighted function

$$
w(t)=\frac{\alpha}{(b-a)^{\alpha}}(b-t)^{\alpha-1}, \quad t \in[a, b],
$$

and hence

$$
W(t)=1-\left(\frac{b-t}{b-a}\right)^{\alpha}, \quad t \in[a, b] .
$$

We obtain

$$
\begin{aligned}
f(x) & =\int_{a}^{b} w(t) f(t) d t+\int_{a}^{b} P_{w}(x, t) f^{\prime}(t) d t \\
& =\frac{\alpha}{(b-a)^{\alpha}} \int_{a}^{b}(b-t)^{\alpha-1} f(t) d t+\int_{a}^{b} P_{w}(x, t) f^{\prime}(t) d t \\
& =\frac{\alpha \Gamma(\alpha)}{(b-a)^{\alpha}}{ }_{a} J_{b}^{\alpha} f(b)+\int_{a}^{b} P_{w}(x, t) f^{\prime}(t) d t
\end{aligned}
$$

Since $\Gamma(\alpha+1)=\alpha \Gamma(\alpha)$ and $P_{w}(x, t)$ for $W(t)=1-$ $((b-t) /(b-a))^{\alpha}$ reduces to $P_{3}(x, t)$, the proof follows. 
Remark 4. In case $\alpha=1$, (12) reduces to Montgomery identity (3).

\section{Montgomery Identity for Fractional Integral of a Function $f$ with respect to Another Function $g$}

Let $\alpha>0, g$ an increasing function on $(a, b]$, and $g^{\prime}$ a continuous function on $(a, b)$. The fractional integral of a function $f$ with respect to another function $g$ is given by

$$
{ }_{a} J_{x ; g}^{\alpha} f(x)=\frac{1}{\Gamma(\alpha)} \int_{a}^{x}(g(x)-g(t))^{\alpha-1} g^{\prime}(t) f(t) d t .
$$

In case $g(x)=x, x \in[a, b],{ }_{a} J_{x ; g}^{\alpha} f(x)$ reduces to the Riemann-Liouville integral operator ${ }_{a} J_{x}^{\alpha} f(x)$.

Theorem 5. Suppose that all the assumptions of Theorem 3 hold. Additionally, assume that $g$ is an increasing function on $(a, b]$ and $g^{\prime}$ a continuous function on $(a, b)$. Then, the following identity holds:

$$
f(x)=\frac{\Gamma(\alpha+1)}{(g(b)-g(a))^{\alpha}} J_{b ; g}^{\alpha} f(b)+\int_{a}^{b} P_{4}(x, t) f^{\prime}(t) d t,
$$

where

$$
\begin{aligned}
& P_{4}(x, t)= \begin{cases}1-\left(\frac{g(b)-g(t)}{g(b)-g(a)}\right)^{\alpha} & a \leq t \leq x, \\
-\left(\frac{g(b)-g(t)}{g(b)-g(a)}\right)^{\alpha} & x<t \leq b,\end{cases} \\
& { }_{a} J_{x ; g}^{\alpha} f(x)=\frac{1}{\Gamma(\alpha)} \int_{a}^{x}(g(x)-g(t))^{\alpha-1} g^{\prime}(t) f(t) d t
\end{aligned}
$$

is the fractional integral of order $\alpha>0$ of a function $f$ with respect to another function $\mathrm{g}$.

Proof. Apply (10) with normalized weighted function

$$
w(t)=\frac{\alpha}{(g(b)-g(a))^{\alpha}}(g(b)-g(t))^{\alpha-1} g^{\prime}(t), \quad t \in[a, b],
$$

and hence

$$
W(t)=1-\left(\frac{g(b)-g(t)}{g(b)-g(a)}\right)^{\alpha}, \quad t \in[a, b] .
$$

We obtain

$$
\begin{aligned}
f(x) & =\int_{a}^{b} w(t) f(t) d t+\int_{a}^{b} P_{w}(x, t) f^{\prime}(t) d t \\
& =\frac{\alpha}{(g(b)-g(a))^{\alpha}} \int_{a}^{b}(g(b)-g(t))^{\alpha-1} g^{\prime}(t) f(t) d t
\end{aligned}
$$

$$
\begin{aligned}
& +\int_{a}^{b} P_{w}(x, t) f^{\prime}(t) d t \\
= & \frac{\alpha \Gamma(\alpha)}{(g(b)-g(a))^{\alpha}} J_{b ; g}^{\alpha} f(b)+\int_{a}^{b} P_{w}(x, t) f^{\prime}(t) d t .
\end{aligned}
$$

Since $P_{w}(x, t)$ for $W(t)=1-((g(b)-g(t)) /(g(b)-g(a)))^{\alpha}$ reduces to $P_{4}(x, t)$, the proof follows.

Remark 6. If we apply (18) with identity function $g(x)=x$, $x \in[a, b]$, (18) reduces to (12).

\section{New Ostrowski Type Inequalities}

In this section, using the Montgomery identity for fractional integrals, we give the Ostrowski inequality for fractional integrals for functions whose first derivatives belong to $L_{p}$ spaces.

Here and hereafter, the symbol $L_{p}[a, b](p \geq 1)$ denotes the space of $p$-power integrable functions on the interval $[a, b]$ equipped with the norm $\|f\|_{p,[a, b]}$ (or simply $\|f\|_{p}$ ) defined by

$$
\|f\|_{p}=\left(\int_{a}^{b}|f(t)|^{p} d t\right)^{1 / p}
$$

and $L_{\infty}[a, b]$ denotes the space of essentially bounded functions on $[a, b]$ with the norm

$$
\|f\|_{\infty}=\operatorname{ess} \sup _{t \in[a, b]}|f(t)|
$$

Theorem 7. Suppose that all the assumptions of Theorem 5 hold. Additionally, assume that $(p, q)$ is a pair of conjugate exponents; that is, $1 \leq p, q \leq \infty,(1 / p)+(1 / q)=1$, and $f^{\prime} \in L_{p}[a, b]$. Then, the following inequality holds:

$$
\left|f(x)-\frac{\Gamma(\alpha+1)}{(g(b)-g(a))^{\alpha}} a_{b ; g}^{\alpha} f(b)\right| \leq\left\|P_{4}(x, t)\right\|_{q}\left\|f^{\prime}\right\|_{p^{\prime}}
$$

where the q-norm is calculated with respect to variable $t$. The constant $\left\|P_{4}(x, t)\right\|_{q}$ is sharp for $1<p \leq \infty$ and the best possible for $p=1$.

Proof. We use the identity (18) and apply the Hölder inequality to get estimate:

$$
\begin{aligned}
\left|f(x)-\frac{\Gamma(\alpha+1)}{(b-a)^{\alpha}}{ }_{a} J_{b ; g}^{\alpha} f(b)\right| & =\left|\int_{a}^{b} P_{4}(x, t) f^{\prime}(t) d t\right| \\
& \leq\left\|P_{4}(x, t)\right\|_{q}\left\|f^{\prime}\right\|_{p} .
\end{aligned}
$$


Let us denote $C^{x}(t)=P_{4}(x, t), t \in[a, b]$. Now, we will prove that the constant $\left\|P_{4}(x, t)\right\|_{q}$ is optimal. For $1<p<\infty$, we will find a function $f$ such that

$$
\begin{aligned}
& \left|\int_{a}^{b} C^{x}(t) f^{\prime}(t) d t\right| \\
& \quad=\left(\int_{a}^{b}\left|C^{x}(t)\right|^{q} d t\right)^{1 / q}\left(\int_{a}^{b}\left|f^{\prime}(t)\right|^{p} d t\right)^{1 / p} .
\end{aligned}
$$

In this case, let $f$ be such that

$$
f^{\prime}(t)=\operatorname{sgn} C^{x}(t) \cdot\left|C^{x}(t)\right|^{1 /(p-1)} .
$$

For $p=\infty$, let

$$
f^{\prime}(t)=\operatorname{sgn} C^{x}(t) .
$$

It remains to prove for $p=1$ that

$$
\left|\int_{a}^{b} C^{x}(t) f^{\prime}(t) d t\right| \leq \sup _{t \in[a, b]}\left|C^{x}(t)\right|\left(\int_{a}^{b}\left|f^{\prime}(t)\right| d t\right)
$$

is the best possible inequality.

Function $C^{x}(t)$ is left continuous on $[a, b]$; more precisely, $C^{x}(t)$ is continuous and increasing on $[a, x]$ and on $(x, b]$ and has a finite jump of -1 at $x$. Also, $C^{x}(a)=C^{x}(b)=0$. Thus, we have two possibilities.

(1) $\left|C^{x}(t)\right|$ attains its maximum at $t_{0} \in(a, b]$. Then, $t_{0}=x$ and $C^{x}\left(t_{0}\right)>0$. For $\varepsilon>0$ small enough, define $f_{\varepsilon}(t)$ by

$$
f_{\varepsilon}(t)= \begin{cases}0, & a \leq t \leq t_{0}-\varepsilon \\ \frac{1}{\varepsilon}\left(t-t_{0}+\varepsilon\right), & t_{0}-\varepsilon \leq t \leq t_{0} \\ 1, & t_{0} \leq t \leq b\end{cases}
$$

Hence,

$$
\left|\int_{a}^{b} C^{x}(t) f_{\varepsilon}^{\prime}(t) d t\right|=\left|\int_{t_{0}-\varepsilon}^{t_{0}} C^{x}(t) \frac{1}{\varepsilon} d t\right|=\frac{1}{\varepsilon} \int_{t_{0}-\varepsilon}^{t_{0}} C^{x}(t) d t .
$$

Now, from inequality (30), we have

$$
\frac{1}{\varepsilon} \int_{t_{0}-\varepsilon}^{t_{0}} C^{x}(t) d t \leq \frac{1}{\varepsilon} C^{x}\left(t_{0}\right) \int_{t_{0}-\varepsilon}^{t_{0}} d t=C^{x}\left(t_{0}\right) .
$$

Since

$$
\lim _{\substack{\varepsilon>0 \\ \varepsilon>0}} \frac{1}{\varepsilon} \int_{t_{0}-\varepsilon}^{t_{0}} C^{x}(t) d t=C^{x}\left(t_{0}\right)
$$

the statement follows.

(2) $\left|C^{x}(t)\right|$ does not attain a maximum on the $[a, b]$ and let $t_{0} \in[a, b]$ be such that

$$
\sup _{t \in[a, b]}\left|C^{x}(t)\right|=\lim _{\substack{\varepsilon \rightarrow 0 \\ \varepsilon>0}}\left|C^{x}\left(t_{0}+\varepsilon\right)\right| .
$$

Then, $t_{0}=x$ and $\lim _{\substack{\varepsilon>0 \\ \varepsilon>0}} C^{x}\left(t_{0}+\varepsilon\right)<0$. Now, we take

$$
f_{\varepsilon}(t)= \begin{cases}1, & a \leq t \leq t_{0}, \\ -\frac{1}{\varepsilon}\left(t-t_{0}-\varepsilon\right), & t_{0} \leq t \leq t_{0}+\varepsilon, \\ 0, & t_{0}+\varepsilon \leq t \leq b,\end{cases}
$$

and similarly as before we have

$$
\begin{aligned}
\left|\int_{a}^{b} C^{x}(t) f_{\varepsilon}^{\prime}(t) d t\right| & =\left|\int_{t_{0}}^{t_{0}+\varepsilon} C^{x}(t)\left(-\frac{1}{\varepsilon}\right) d t\right| \\
& =\frac{1}{\varepsilon} \int_{t_{0}}^{t_{0}+\varepsilon}\left|C^{x}(t)\right| d t,
\end{aligned}
$$

$$
\frac{1}{\varepsilon} \int_{t_{0}}^{t_{0}+\varepsilon}\left|C^{x}(t)\right| d t \leq \frac{1}{\varepsilon}\left|C^{x}(t)\right| \int_{t_{0}}^{t_{0}+\varepsilon} d t=\left|C^{x}\left(t_{0}\right)\right|,
$$

$$
\lim _{\varepsilon \rightarrow 0} \frac{1}{\varepsilon} \int_{t_{0}}^{t_{0}+\varepsilon}\left|C^{x}(t)\right| d t=\left|C^{x}\left(t_{0}\right)\right|
$$

and the proof is completed.

Corollary 8. Suppose that all the assumptions of Theorem 7 hold. Then, the following inequality holds:

$$
\left|f(x)-\frac{\Gamma(\alpha+1)}{(b-a)^{\alpha}}{ }_{a} J_{b}^{\alpha} f(b)\right| \leq\left\|P_{3}(x, t)\right\|_{q}\left\|f^{\prime}\right\|_{p^{\prime}}
$$

where the q-norm is calculated with respect to variable t. The constant $\left\|P_{3}(x, t)\right\|_{q}$ is sharp for $1<p \leq \infty$ and the best possible for $p=1$.

Proof. We apply Theorem 7 with identity function $g(x)=x$, $x \in[a, b]$, and the proof follows directly.

Theorem 9. Suppose that all the assumptions of Theorem 7 hold. Then, for $1<p \leq \infty$, the following inequality holds:

$$
\begin{aligned}
\mid f(x) & -\frac{\Gamma(\alpha+1)}{(g(b)-g(a))^{\alpha}} J_{b ; g}^{\alpha} f(b) \mid \\
\leq & {\left[\left(1-\left(\frac{g(b)-g(x)}{g(b)-g(a)}\right)^{\alpha}\right)^{q}(x-a)\right.} \\
& \left.+\left(\left(\frac{g(b)-g(x)}{g(b)-g(a)}\right)^{\alpha}\right)^{q}(b-x)\right]^{1 / q}\left\|f^{\prime}\right\|_{p},
\end{aligned}
$$

while for $p=1$

$$
\begin{aligned}
& \left|f(x)-\frac{\Gamma(\alpha+1)}{(g(b)-g(a))^{\alpha}}{ }_{a} J_{b ; g}^{\alpha} f(b)\right| \\
& \quad \leq\left[\frac{1}{2}+\left|\frac{1}{2}-\left(\frac{g(b)-g(x)}{g(b)-g(a)}\right)^{\alpha}\right|\right]\left\|f^{\prime}\right\|_{1} .
\end{aligned}
$$


Proof. We use the identity (18) and apply the Hölder inequality to get estimate:

$$
\begin{aligned}
\mid f & (x)-\frac{\Gamma(\alpha+1)}{(b-a)^{\alpha}}{ }_{a} J_{b ; g}^{\alpha} f(b) \mid \\
= & \left|\int_{a}^{b} P_{4}(x, t) f^{\prime}(t) d t\right| \\
\leq & \left|\int_{a}^{x} P_{4}(x, t) f^{\prime}(t) d t\right|+\left|\int_{x}^{b} P_{4}(x, t) f^{\prime}(t) d t\right| \\
\leq & \left\|P_{4}(x, t)\right\|_{q,[a, x]}\left\|f^{\prime}\right\|_{p,[a, x]}+\left\|P_{4}(x, t)\right\|_{q,[x, b]}\left\|f^{\prime}\right\|_{p,[x, b]} \\
= & \left\|1-\left(\frac{g(b)-g(t)}{g(b)-g(a)}\right)^{\alpha}\right\|_{q,[a, x]}\left\|f^{\prime}\right\|_{p,[a, x]} \\
& +\left\|-\left(\frac{g(b)-g(t)}{g(b)-g(a)}\right)^{\alpha}\right\|_{q,[x, b]}\left\|f^{\prime}\right\|_{p,[x, b]} .
\end{aligned}
$$

Since $1-((g(b)-g(t)) /(g(b)-g(a)))^{\alpha}$ is increasing on $[a, x]$ and $((g(b)-g(t)) /(g(b)-g(a)))^{\alpha}$ is decreasing on $[x, b]$, in case $1<p, q<\infty$, we have

$$
\begin{aligned}
\| 1 & -\left(\frac{g(b)-g(t)}{g(b)-g(a)}\right)^{\alpha} \|_{q,[a, x]} \\
& \leq\left(1-\left(\frac{g(b)-g(x)}{g(b)-g(a)}\right)^{\alpha}\right)(x-a)^{1 / q}, \\
\|- & \left(\frac{g(b)-g(t)}{g(b)-g(a)}\right)^{\alpha} \|_{q,[x, b]} \\
& \leq\left(\frac{g(b)-g(x)}{g(b)-g(a)}\right)^{\alpha}(b-x)^{1 / q} .
\end{aligned}
$$

Now, apply discrete Hölder inequality to get

$$
\begin{aligned}
\| 1- & \left(\frac{g(b)-g(t)}{g(b)-g(a)}\right)^{\alpha}\left\|_{q,[a, x]}\right\| f^{\prime} \|_{p,[a, x]} \\
& +\left\|-\left(\frac{g(b)-g(t)}{g(b)-g(a)}\right)^{\alpha}\right\|_{q,[x, b]}\left\|f^{\prime}\right\|_{p,[x, b]} \\
\leq & \left(1-\left(\frac{g(b)-g(x)}{g(b)-g(a)}\right)^{\alpha}\right)(x-a)^{1 / q}\left\|f^{\prime}\right\|_{p,[a, x]} \\
& +\left(\frac{g(b)-g(x)}{g(b)-g(a)}\right)^{\alpha}(b-x)^{1 / q}\left\|f^{\prime}\right\|_{p,[x, b]} \\
\leq & {\left[\left(1-\left(\frac{g(b)-g(x)}{g(b)-g(a)}\right)^{\alpha}\right)^{q}(x-a)\right.} \\
& \left.+\left(\left(\frac{g(b)-g(x)}{g(b)-g(a)}\right)^{\alpha}\right)^{q}(b-x)\right]^{1 / q} \\
& \cdot\left[\left(\left\|f^{\prime}\right\|_{p,[a, x]}\right)^{p}+\left(\left\|f^{\prime}\right\|_{p,[x, b]}\right)^{p}\right]^{1 / p}
\end{aligned}
$$

$$
\begin{aligned}
=\left[\left(1-\left(\frac{g(b)-g(x)}{g(b)-g(a)}\right)^{\alpha}\right)^{q}(x-a)\right. \\
\left.\quad+\left(\left(\frac{g(b)-g(x)}{g(b)-g(a)}\right)^{\alpha}\right)^{q}(b-x)\right]^{1 / q}\left\|f^{\prime}\right\|_{p,[a, b]}
\end{aligned}
$$

The proof is similar to $p=\infty$, while for $p=1$ we have

$$
\begin{aligned}
\| 1- & \left(\frac{g(b)-g(t)}{g(b)-g(a)}\right)^{\alpha}\left\|_{\infty,[a, x]}\right\| f^{\prime} \|_{1,[a, x]} \\
& +\left\|-\left(\frac{g(b)-g(t)}{g(b)-g(a)}\right)^{\alpha}\right\|_{\infty,[x, b]}\left\|f^{\prime}\right\|_{1,[x, b]} \\
\leq & \max \left\{\left(1-\left(\frac{g(b)-g(x)}{g(b)-g(a)}\right)^{\alpha}\right),\left(\frac{g(b)-g(x)}{g(b)-g(a)}\right)^{\alpha}\right\} \\
& \times\left(\left\|f^{\prime}\right\|_{1,[a, x]}+\left\|f^{\prime}\right\|_{1,[x, b]}\right) \\
= & {\left[\frac{1}{2}+\mid \frac{1}{2}-\left(\frac{g(b)-g(x)}{g(b)-g(a)}\right)^{\alpha} \|\right]\left\|f^{\prime}\right\|_{1} . }
\end{aligned}
$$

In the last line, the identity $\max \{A, B\}=(A+B) / 2+|(A-B) / 2|$ was used.

Corollary 10. Suppose that all the assumptions of Theorem 7 hold. Then, for $1<p \leq \infty$, the following inequality holds:

$$
\begin{aligned}
\mid f(x) & -\frac{\Gamma(\alpha+1)}{(b-a)^{\alpha}}{ }_{a} J_{b}^{\alpha} f(b) \mid \\
\leq & {\left[\left(1-\left(\frac{b-x}{b-a}\right)^{\alpha}\right)^{q}(x-a)\right.} \\
& \left.+\left(\left(\frac{b-x}{b-a}\right)^{\alpha}\right)^{q}(b-x)\right]^{1 / q}\left\|f^{\prime}\right\|_{p}
\end{aligned}
$$

while for $p=1$

$$
\left|f(x)-\frac{\Gamma(\alpha+1)}{(b-a)^{\alpha}}{ }_{a} J_{b}^{\alpha} f(b)\right| \leq\left[\frac{1}{2}+\left|\frac{1}{2}-\left(\frac{b-x}{b-a}\right)^{\alpha}\right|\right]\left\|f^{\prime}\right\|_{1} .
$$

Proof. We apply Theorem 9 with identity function $g(x)=x$, $x \in[a, b]$, and the proof follows directly.

\section{Application for Hadamard Fractional Integrals}

Hadamard fractional integrals of order $\alpha>0$ for $0<a<x$ are given by

$$
{ }_{a} H_{x}^{\alpha} f(x)=\frac{1}{\Gamma(\alpha)} \int_{a}^{x}\left(\ln \frac{x}{y}\right)^{\alpha-1} \frac{f(y)}{y} d y .
$$

Notice that Hadamard fractional integrals of order $\alpha$ are special case of fractional integrals with respect to another function $g$, when $g(x)=\ln x, x \in[a, b]$. 
The following result is a weighted Ostrowski type result for Hadamard fractional integrals of order $\alpha$.

Corollary 11. Suppose that all the assumptions of Theorem 7 and $0<a<x$ hold. Then, for $1<p \leq \infty$, the following inequality holds:

$$
\begin{aligned}
\mid f(x) & -\frac{\Gamma(\alpha+1)}{(\ln (b / a))^{\alpha}}{ }_{a} H_{b}^{\alpha} f(b) \mid \\
\leq & {\left[\left(\left\|1-\left(\frac{\ln (b / t)}{\ln (b / a)}\right)^{\alpha}\right\|_{q,[a, x]}\right)^{q}\right.} \\
& \left.+\left(\left\|\left(\frac{\ln (b / t)}{\ln (b / a)}\right)^{\alpha}\right\|_{q,[x, b]}\right)^{q}\right]^{1 / q}\left\|f^{\prime}\right\|_{p} \\
\leq & {\left[\left(1-\left(\frac{\ln (b / x)}{\ln (b / a)}\right)^{\alpha}\right)^{q}(x-a)\right.} \\
& \left.+\left(\left(\frac{\ln (b / x)}{\ln (b / a)}\right)^{\alpha}\right)^{q}(b-x)\right]^{1 / q}\left\|f^{\prime}\right\|_{p},
\end{aligned}
$$

where the q-norm is calculated with respect to variable $t$, while for $p=1$

$$
\begin{aligned}
& \left|f(x)-\frac{\Gamma(\alpha+1)}{(\ln (b / a))^{\alpha}}{ }_{a} H_{b}^{\alpha} f(b)\right| \\
& \quad \leq\left[\frac{1}{2}+\left|\frac{1}{2}-\left(\frac{\ln (b / x)}{\ln (b / a)}\right)^{\alpha}\right|\right]\left\|f^{\prime}\right\|_{1} .
\end{aligned}
$$

The constant in the first inequality for $1<p \leq \infty$ is sharp and the best possible for $p=1$.

Proof. We apply Theorems 7 and 9 with $g(x)=\ln x, x \in$ $[a, b]$.

\section{Conflict of Interests}

The author declares that there is no conflict of interests regarding the publication of this paper.

\section{Acknowledgment}

This work has been fully supported by Croatian Science Foundation under the Project 5435.

\section{References}

[1] A. Ostrowski, "Über die Absolutabweichung einer differentiebaren Funktion von ihrem Integralmittelwert," Commentarii Mathematici Helvetici, vol. 10, no. 1, pp. 226-227, 1937.

[2] D. S. Mitrinović, J. E. Pečarić, and A. M. Fink, Inequalities for Functions and their Integrals and Derivatives, Kluwer Academic, Dordrecht, The Netherlands, 1994.

[3] G. Anastassiou, M. R. Hooshmandasl, A. Ghasemi, and F. Moftakharzadeh, "Montgomery identities for fractional integrals and related fractional inequalities," Journal of Inequalities in Pure and Applied Mathematics, vol. 10, no. 4, article 97, 2009.
[4] M. Z. Sarikaya and H. Ogunmez, "On new inequalities via Riemann-Liouville fractional integration," Abstract and Applied Analysis, vol. 2012, Article ID 428983, 10 pages, 2012.

[5] A. Aglić Aljinović, M. Krnić, and J. Pečarić, "Weighted montgomery identity for fractional integral of a function with respect to another function," Georgian Mathematical Journal, vol. 21, no. 1, pp. 1-10, 2014.

[6] J. Pečarić, "On the Čebyšev inequality," Buletinul Ştiinţific şi Tehnic Institutului Politehnic "Traian Vuia” Timişoara, vol. 25, no. 39, pp. 5-9, 1980. 


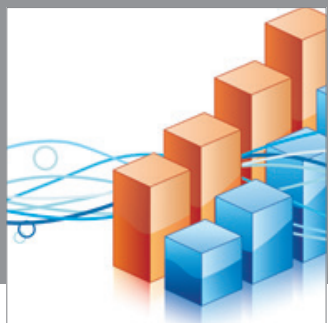

Advances in

Operations Research

mansans

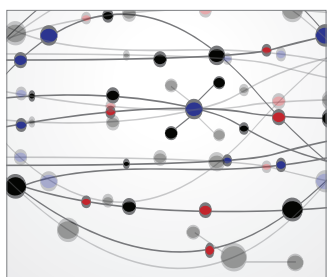

The Scientific World Journal
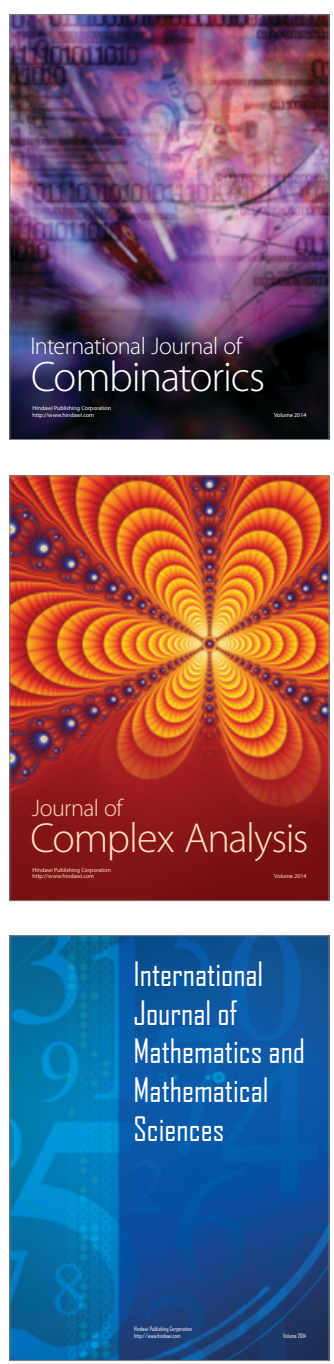
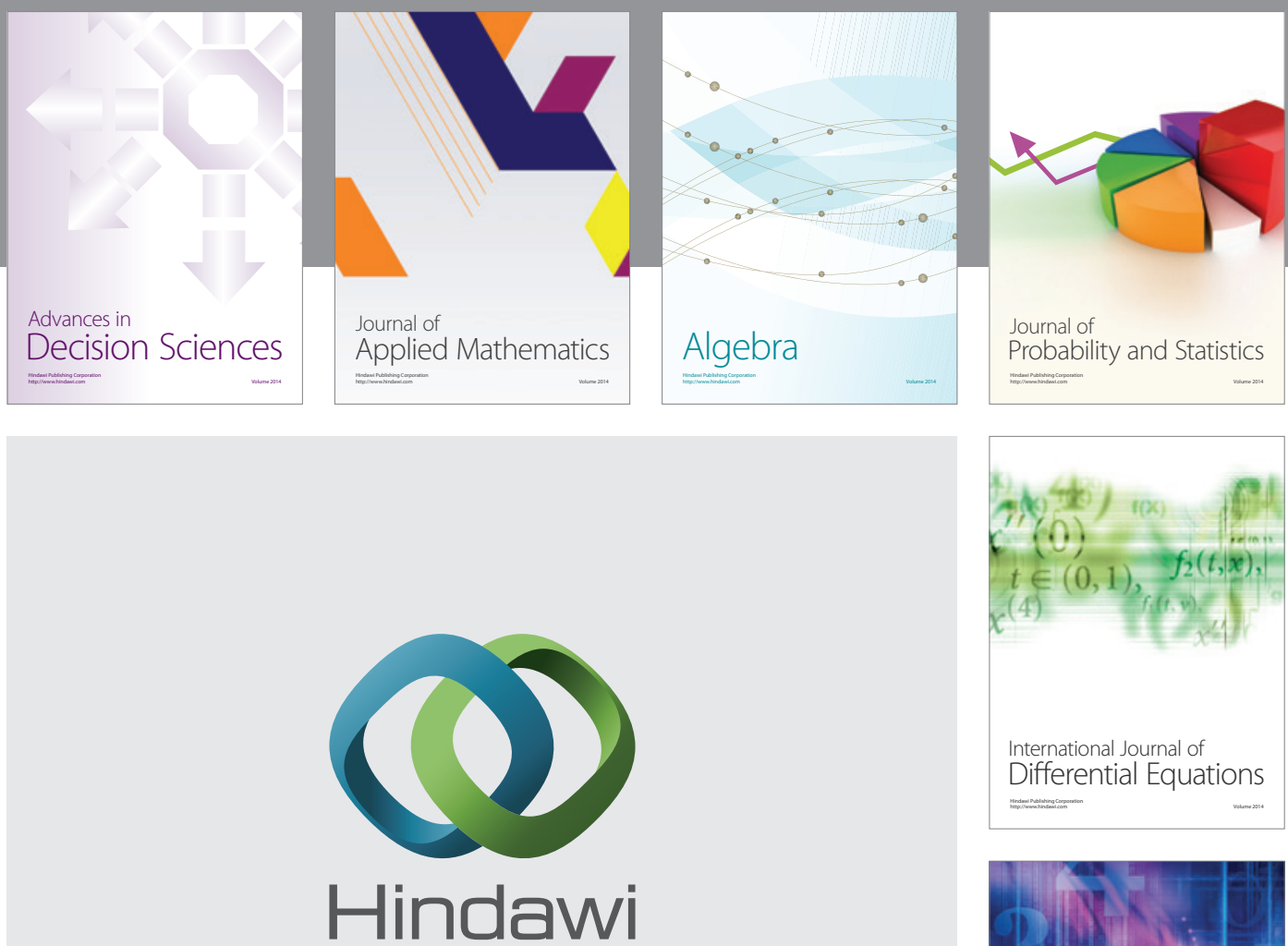

Submit your manuscripts at http://www.hindawi.com
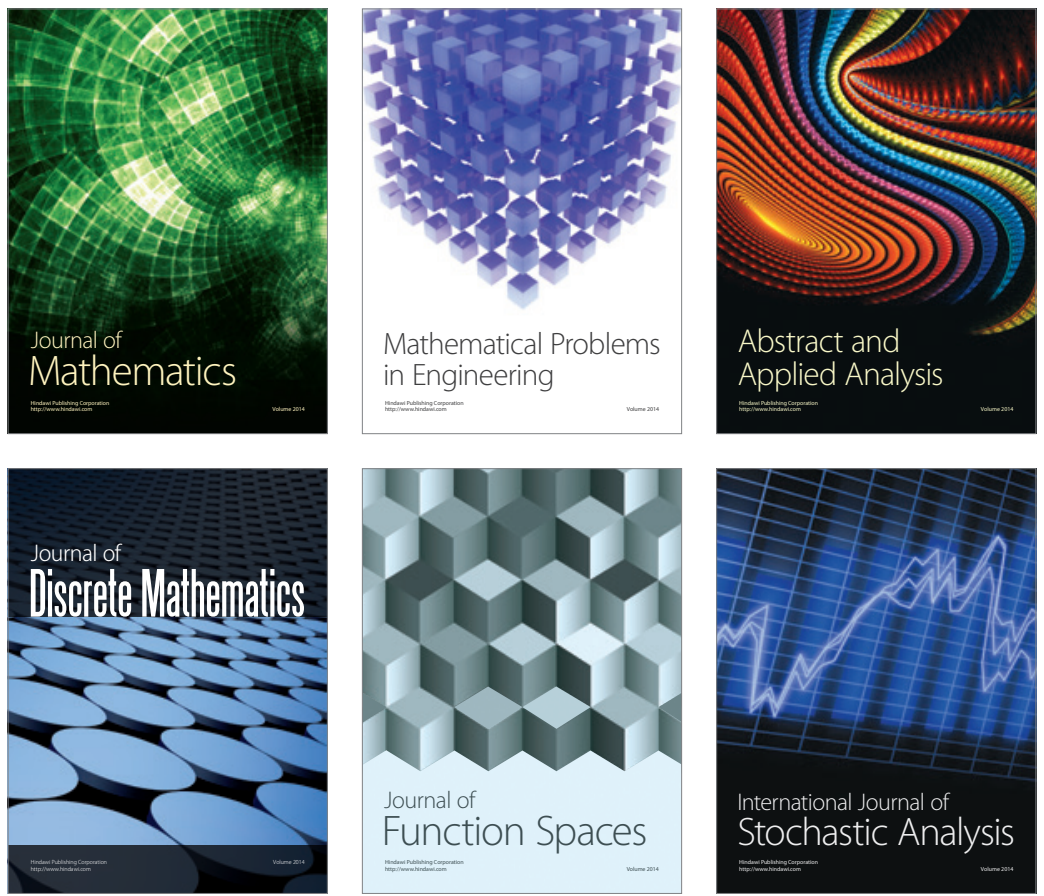

Journal of

Function Spaces

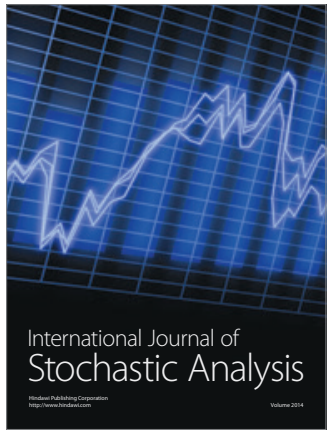

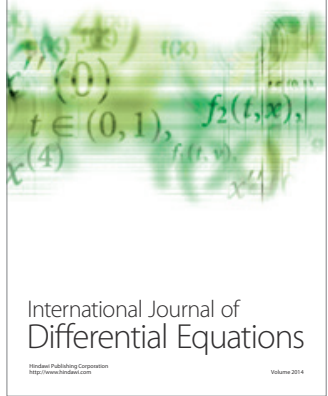
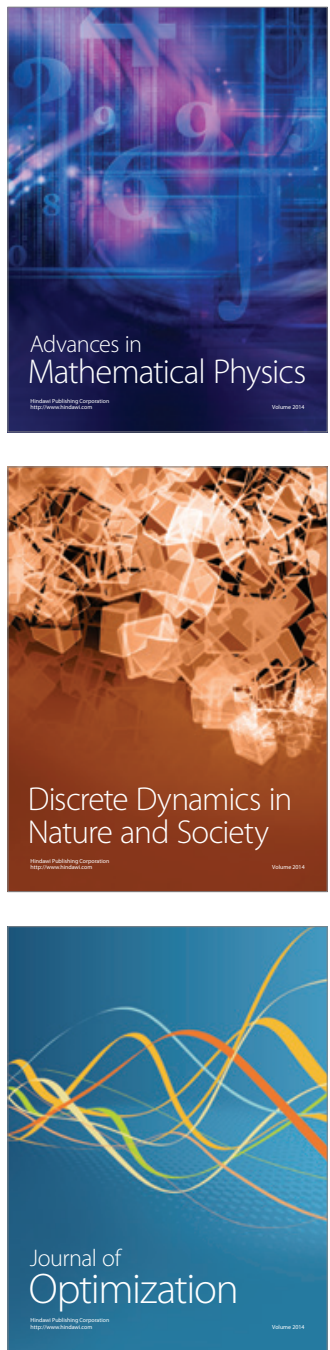